Received by the editors: December 04, 2019; Accepted:June 15, 2020

\title{
SOME NUMERICAL CHARACTERISTICS OF DIRECT SUM OF OPERATORS
}

\author{
Elif OTKUN ÇEVİK
}

Avrasya University, Trabzon, TURKEY

\begin{abstract}
The connection between some numerical characteristics (numerical range, numerical radius, Crawford number and sectoriality) of direct sum of operators in the direct sum of Hilbert spaces and their coordinate operators has been investigated.
\end{abstract}

\section{INTRODUCTION}

The general information on numerical characteristics (as numerical range, numerical radius, Crawford number, sectoriality and etc.) can be found in [1-7]. The obtained results may be applied in perturbation theory, generalized eigenvalue problems, numerical analysis, system theory, dilation theory and etc. [1-4].

It is known that infinite direct sum of Hilbert spaces $H_{n}, n \geq 1$ and infinite direct sum of operators $A_{n}$ in $H_{n}, n \geq 1$ are defined as

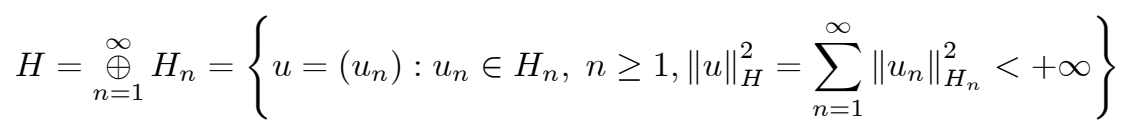

and

$$
\begin{gathered}
A=\bigoplus_{n=1}^{\infty} A_{n}, D(A)=\left\{u=\left(u_{n}\right) \in H: u_{n} \in D\left(A_{n}\right), n \geq 1, A u=\left(A_{n} u_{n}\right) \in H\right\}, \\
A: D(A) \subset H \rightarrow H
\end{gathered}
$$

(see $[8])$.

2020 Mathematics Subject Classification. Primary 47A12, 47B44; Secondary 46C05.

Keywords and phrases. Direct sum of operators, numerical range, numerical radius, Crawford number, sectorial operator.

elif.ocevik@avrasya.edu.tr

(D) 0000-0001-8506-1889.

(C) 2020 Ankara University Communications Faculty of Sciences University of Ankara-Series A1 Mathematics and Statistics 
The general theory of linear closed operators in Hilbert spaces and its applications to physical problems has been investigated by many mathematicians (see for example [8]).

However, many physical problems of today arising in the modelling of processes of multi-particle quantum mechanics, quantum field theory and in the physics of rigid bodies support to study a theory of linear direct sum of operators in the direct sum of Hilbert spaces (see [9-11] and references there in).

Connections between numerical range and numerical radius for the direct sum of two operators in the direct sum of Hilbert spaces and coordinate operators have been investigated in [6].

In this work, the connection between some numerical characteristics of direct sum of operators in direct sum of Hilbert spaces and their coordinate operators will be investigated.

\section{Numerical Range of Direct Sum Operators}

Definition 1. [1] Let $\mathcal{H}$ be a Hilbert space with inner product $(\cdot, \cdot)$ and norm $\|\cdot\|$ be induced norm by this inner product. In this case, the numerical range of a linear bounded operator $T$ in $\mathcal{H}$ is the subset of the complex numbers $\mathbb{C}$ given by

$$
W(T)=\{(T x, x) \in \mathbb{C}: x \in \mathcal{H},\|x\|=1\} .
$$

Recall that a numerical range of an operator is convex (Toeplitz-Housdorff) and spectrum of an operator is contained in the closure of its numerical range.

The following result is true.

Theorem 2. If for any $n \geq 1, H_{n}$ is a Hilbert space, $A_{n} \in L\left(H_{n}\right), H=\bigoplus_{n=1}^{\infty} H_{n}$ and $A=\bigoplus_{n=1}^{\infty} A_{n}, A \in L(H)$, then numerical range of the operator $A$ is in the form

$$
W(A)=c o\left(\bigcup_{n=1}^{\infty} W\left(A_{n}\right)\right),
$$

where co $(\Omega), \Omega \subset \mathbb{C}$ denotes the convex hull of $\Omega$.

Proof. Indeed in this case, for any element $f \in H$ with norm

$$
\|f\|_{H}^{2}=\sum_{n=1}^{\infty}\left\|f_{n}\right\|_{n}^{2}=1, f_{n} \neq 0, n \geq 1,
$$

we have

$$
(A f, f)_{H}=\sum_{n=1}^{\infty}\left(A_{n} f_{n}, f_{n}\right)_{n}=\sum_{n=1}^{\infty}\left\|f_{n}\right\|_{n}^{2}\left(A_{n}\left(f_{n} /\left\|f_{n}\right\|_{n}\right), f_{n} /\left\|f_{n}\right\|_{n}\right)_{n}=\sum_{n=1}^{\infty} \alpha_{n} \mu_{n},
$$

where

$$
\alpha_{n}=\left\|f_{n}\right\|_{n}^{2}, \mu_{n}=\left(A_{n}\left(f_{n} /\left\|f_{n}\right\|_{n}\right), f_{n} /\left\|f_{n}\right\|_{n}\right)_{n} \in W\left(A_{n}\right), n \geq 1 .
$$


It is clear that $\sum_{n=1}^{\infty} \alpha_{n}=1$.

Now, assume that there exists a number $n_{k} \in \mathbb{N} \cup\{+\infty\}$ such that

$$
f_{n_{1}} \neq 0, f_{n_{2}} \neq 0, \ldots, f_{n_{k}} \neq 0, k \leq \infty
$$

and

$$
f=\left(0, \ldots, 0, f_{n_{1}}, 0, \ldots, 0, f_{n_{2}}, 0, \ldots, 0, f_{n_{k}}, 0, \ldots, 0, \ldots\right) .
$$

In this case,

$$
\begin{aligned}
(A f, f)_{H} & =\sum_{j=1}^{k}\left(A_{n_{j}} f_{n_{j}}, f_{n_{j}}\right)_{n_{j}} \\
& =\sum_{j=1}^{k}\left\|f_{n_{j}}\right\|_{n_{j}}^{2}\left(A_{n_{j}}\left(f_{n_{j}} /\left\|f_{n_{j}}\right\|_{n_{j}}\right), f_{n_{j}} /\left\|f_{n_{j}}\right\|_{n_{j}}\right)_{n_{j}} \\
& =\sum_{j=1}^{k} \alpha_{n_{j}} \mu_{n_{j}}
\end{aligned}
$$

where

$$
\alpha_{n_{j}}=\left\|f_{n_{j}}\right\|_{n_{j}}^{2}, \mu_{n_{j}}=\left(A_{n_{j}}\left(f_{n_{j}} /\left\|f_{n_{j}}\right\|_{n_{j}}\right), f_{n_{j}} /\left\|f_{n_{j}}\right\|_{n_{j}}\right)_{n_{j}} \in W\left(A_{n_{j}}\right) .
$$

It is clear that $\sum_{j=1}^{k} \alpha_{n_{j}}=1$.

\section{Numerical Radius of Direct Sum Operators}

Definition 3. [1] Let $\mathcal{H}$ be a Hilbert space with inner product $(\cdot, \cdot)$ and norm $\|\cdot\|$ be induced norm by this inner product. In this case, the numerical radius of a linear bounded operator $T$ in $\mathcal{H}$ is a number which is given by

$$
w(T)=\sup \{|\lambda|: \lambda \in W(T)\} .
$$

Recall that for any vector $x \in \mathcal{H}$, it is true that

$$
|(T x, x)| \leq w(T)\|x\|^{2} .
$$

The following result is true.

Theorem 4. If for any $n \geq 1, H_{n}$ is a Hilbert space, $A_{n} \in L\left(H_{n}\right), H=\bigoplus_{n=1}^{\infty} H_{n}$ and $A=\bigoplus_{n=1}^{\infty} A_{n}, A \in L(H)$, then numerical radius of the operator $A$ is in the following form

$$
w(A)=\sup _{n \geq 1} w\left(A_{n}\right)
$$


Proof. For any $n \geq 1$, since $w\left(A_{n}\right) \leq w(A)$, then

$$
\sup _{n \geq 1} w\left(A_{n}\right) \leq w(A) \text {. }
$$

On the other hand, if $\mu \in W(A)$, then $\mu=\sum_{n=1}^{\infty} \alpha_{n} \mu_{n}, \sum_{n=1}^{\infty} \alpha_{n}=1$ and $\mu_{n} \in$ $W\left(A_{n}\right)$ for $n \geq 1$. Then

$$
|\mu|=\left|\sum_{n=1}^{\infty} \alpha_{n} \mu_{n}\right| \leq \sum_{n=1}^{\infty} \alpha_{n}\left|\mu_{n}\right| \leq \sum_{n=1}^{\infty} \alpha_{n} w\left(A_{n}\right) \leq \sup _{n \geq 1} w\left(A_{n}\right) \sum_{n=1}^{\infty} \alpha_{n}=\sup _{n \geq 1} w\left(A_{n}\right) .
$$

Hence, by the definition of numerical radius we get

$$
w(A) \leq \sup _{n \geq 1} w\left(A_{n}\right) .
$$

Combining two inequalities (1) and (2), we reach to the desired inequality.

\section{Crawford Number of Direct Sum Operators}

Definition 5. [1] Let $\mathcal{H}$ be a Hilbert space with inner product $(\cdot, \cdot)$ and norm $\|\cdot\|$ be induced norm by this inner product. In this case, the Crawford number of a linear bounded operator $T$ in $\mathcal{H}$ is given by

$$
c(T)=\inf \{|\lambda|: \lambda \in W(T)\} .
$$

The following results are true.

Theorem 6. If for any $n \geq 1, H_{n}$ is a Hilbert space, $A_{n} \in L\left(H_{n}\right), H=\bigoplus_{n=1}^{\infty} H_{n}$ and $A=\bigoplus_{n=1}^{\infty} A_{n}, A \in L(H)$, then Crawford number of the operator $A$ is in the following form

$$
c(A) \leq \inf _{n \geq 1} c\left(A_{n}\right) .
$$

Proof. Since $c(A) \leq c\left(A_{n}\right)$, for any $n \geq 1, c(A) \leq \inf _{n \geq 1} c\left(A_{n}\right)$ satisfies.

Theorem 7. If for any $n \geq 1, H_{n}$ is a Hilbert space, $A_{n} \in L\left(H_{n}\right), \operatorname{Re}\left(A_{n}\right) \geq 0(\leq$ $0), H=\bigoplus_{n=1}^{\infty} H_{n}$ and $A=\bigoplus_{n=1}^{\infty} A_{n}, A \in L(H)$, then Crawford number of the operator $A$ is in the following form

$$
c(A)=\inf _{n \geq 1} c\left(A_{n}\right)
$$

Proof. If $\mu \in W(A)$, then by Theorem 2, $\mu=\sum_{n=1}^{\infty} \alpha_{n} \mu_{n}, \sum_{n=1}^{\infty} \alpha_{n}=1$ and $\mu_{n} \in$ $W\left(A_{n}\right), n \geq 1$. 
On the other hand, since for any $n \geq 1, \mu_{n} \geq 0(\leq 0)$, we have

$$
|\mu|=\left|\sum_{n=1}^{\infty} \alpha_{n} \mu_{n}\right|=\sum_{n=1}^{\infty} \alpha_{n}\left|\mu_{n}\right| \geq \sum_{n=1}^{\infty} \alpha_{n} c\left(A_{n}\right) \geq \inf _{n \geq 1} c\left(A_{n}\right) \sum_{n=1}^{\infty} \alpha_{n}=\inf _{n \geq 1} c\left(A_{n}\right) .
$$

Therefore,

$$
c(A) \geq \inf _{n \geq 1} c\left(A_{n}\right) .
$$

In this case, equation (3) and Theorem 6 complete the proof of proposition.

\section{Sectoriality of Direct Sum Operators}

Definition 8. [1] Let $T$ be a linear bounded operator in Hilbert space $\mathcal{H}$. If $\operatorname{Re}(T x, x) \geq 0$ for any $x \in \mathcal{H}$, then it is called an accretive operator in $\mathcal{H}$.

Definition 9. [1] Assume that $T \in L(\mathcal{H})$ is a accretive operator. If $W(T) \subset$ $\{z \in \mathbb{C}:|\arg z|<\varphi\}$ for any $\varphi \in[0, \pi / 2)$, then it is called a sectorial operator with vertex $\gamma=0$ and semi-angle $\varphi$. In this case, $T \in S_{\varphi}(\mathcal{H})$.

The following result is true.

Theorem 10. If for any $n \geq 1, H_{n}$ is a Hilbert space, $A_{n} \in L\left(H_{n}\right), A_{n} \in S_{\varphi_{n}}\left(H_{n}\right), H=$ $\bigoplus_{n=1}^{\infty} H_{n}$ and $A=\bigoplus_{n=1}^{\infty} A_{n}, A \in L(H)$, then for some $\varphi \in[0, \pi / 2)$,

$A \in S_{\varphi}(H)$ thenecessaryandsufficientconditionis $\sup _{n \geq 1} \varphi_{n}<\varphi$.

Proof. From Theorem 2, it is clear that $W\left(A_{n}\right) \subset W(A)$ for any $n \geq 1$.

On the other hand, if $A_{n} \in S_{\varphi_{n}}\left(H_{n}\right), n \geq 1$, then for any $x \in H$ with norm

$$
\|x\|_{H}=\left(\sum_{n=1}^{\infty}\|x\|_{n}^{2}\right)^{1 / 2}=1, n \geq 1
$$

we have

$$
\operatorname{Re}(A x, x)=\sum_{n=1}^{\infty} \operatorname{Re}\left(A_{n} x_{n}, x_{n}\right)_{n} \geq 0 .
$$

Then $A: H \rightarrow H$ is an accretive operator in $H$.

Moreover, it is clear that

$$
\bigcup_{n=1}^{\infty}\left\{z \in W\left(A_{n}\right):|\arg z| \leq \varphi_{n}\right\} \subset W(A) .
$$

If we choose $\varphi=\sup _{n \geq 1} \varphi_{n}$, then

$$
W(A) \subset\{z \in \mathbb{C}:|\arg z| \leq \varphi\} .
$$

Since $\varphi \in[0, \pi / 2)$, then $A \in S_{\varphi}(H)$. 
On the contrary, if $A \in S_{\varphi}(H)$ for some $\varphi \in[0, \pi / 2)$, then from the relation $W\left(A_{n}\right) \subset W(A)$ and accretivity of each coordinate operator $A_{n} \in L\left(H_{n}\right), n \geq 1$, we get

$$
W\left(A_{n}\right) \subset\{z \in \mathbb{C}:|\arg z| \leq \varphi\} .
$$

Consequently, for any $n \geq 1, A_{n} \in S_{\varphi_{n}}\left(H_{n}\right)$. This completes the validity of the assertion.

\section{Applications of Some Numerical Characteristics of Direct Sum}

\section{OPERATORS}

Example 11. Let for any $n \geq 1, H_{n}=(\mathbb{C},|\cdot|), A_{n}=\alpha_{n} E, \alpha_{n} \in \mathbb{C}$ and $H=$ $\bigoplus_{n=1}^{\infty} H_{n}=l_{2}(\mathbb{C}), A=\bigoplus_{n=1}^{\infty}\left(\alpha_{n} E\right): H \rightarrow H$. In this case,

$$
\begin{aligned}
W\left(A_{n}\right) & =\alpha_{n}, n \geq 1, \\
w\left(A_{n}\right) & =\left|\alpha_{n}\right|, n \geq 1, \\
c\left(A_{n}\right) & =\left|\alpha_{n}\right|, n \geq 1
\end{aligned}
$$

and for $\operatorname{Re}\left(\alpha_{n}\right) \geq 0, n \geq 1, A_{n}$ is accretive. In addition

$$
\varphi_{n}=\arg _{n}, n \geq 1 \text {. }
$$

Therefore,

$$
\begin{aligned}
W(A) & =\operatorname{co}\left\{\alpha_{n}, n \geq 1\right\}, \\
w(A) & =\sup _{n \geq 1}\left|\alpha_{n}\right|, \\
c(A) & =\inf _{n \geq 1}\left|\alpha_{n}\right|
\end{aligned}
$$

and this case when sup $\left|\arg \alpha_{n}\right|<\pi / 2$, angle of sectoriality of operator $A$ is

$$
\varphi=\sup _{n \geq 1}\left|\arg \alpha_{n}\right| .
$$

i.e. $A \in S_{\varphi}\left(l_{2}\right)$.

Acknowledgment. I thank Prof. Z.I. Ismailov (Department of Mathematics, Karadeniz Technical University, Trabzon/TURKEY) for his advice and enthusiastic support.

\section{REFERENCES}

[1] Gustafson, K.E., Rao, D.K.M., Numerical Range: The Field of Values of Linear Operators and Matrices, Springer, New York, 1997.

[2] Halmos, P., A Hilbert Space Problem Book (sec. ed.), Springer-Verlag, New York, 1974.

[3] Bonsall, F.F., Duncan, J., Studies in Functional Analysis-Numerical Ranges, Stud. Math., vol.21, Mathematical Association of America, 1980.

[4] Kato, T., Perturbation Theory for Linear Operators, Springer-Verlag, New York, 1980. 
[5] Shapiro, J.H., Lecture Notes, Notes on the Numerical Range, 2003.

[6] Goldberg, M., Tadmor, E., On the Numerical Radius and Its Applications, Linear Algebra and Its Applications, 42 (1982), 263-284.

[7] Ando, T., Li, C. K., The Numerical Range and Numerical Radius, Special Issue, Linear and Multilinear Algebra 37 (1994), 1-238.

[8] Dunford, N., Schwartz, J. T., Linear Operators I; II, Second ed., Interscience Publishers, New York, 1958; 1963

[9] Ismailov, Z.I., Multipoint Normal Differential Operators for First Order, Opusc. Math. 29 (4) (2009), 399-414.

[10] Kochubei, A. N., Symmetric Operators and Nonclassical Spectral Problems, Mat. Zametki 25 (3) (1979), 425-434.

[11] Zettl, A., Sturm-Liouville Theory, First ed., Amer. Math. Soc., USA, 2005. 